\title{
Collective non-perturbative coupling of 2D electrons with high-quality-factor terahertz cavity photons
}

\author{
Qi Zhang ${ }^{1}$, Minhan Lou', Xinwei Li ${ }^{1}$, John L. Reno ${ }^{2}$, Wei Pan ${ }^{3}$, John D. Watson ${ }^{4}$, Michael J. Manfra ${ }^{4,5}$ \\ and Junichiro Kono $0^{1,6,7 \star}$
}

The collective interaction of electrons with light in a highquality-factor cavity is expected to reveal new quantum phenomena ${ }^{1-7}$ and find applications in quantum-enabled technologies $^{8,9}$. However, combining a long electronic coherence time, a large dipole moment, and a high quality-factor has proved difficult ${ }^{10-13}$. Here, we achieved these conditions simultaneously in a two-dimensional electron gas in a high-quality-factor terahertz cavity in a magnetic field. The vacuum Rabi splitting of cyclotron resonance exhibited a square-root dependence on the electron density, evidencing collective interaction. This splitting extended even where the detuning is larger than the resonance frequency. Furthermore, we observed a peak shift due to the normally negligible diamagnetic term in the Hamiltonian. Finally, the high-quality-factor cavity suppressed superradiant cyclotron resonance decay, revealing a narrow intrinsic linewidth of $5.6 \mathrm{GHz}$. High-quality-factor terahertz cavities will enable new experiments bridging the traditional disciplines of condensed-matter physics and cavity-based quantum optics.

Strong resonant light-matter coupling in a cavity setting is an essential ingredient in fundamental cavity quantum electrodynamics (QED) studies ${ }^{14}$ as well as in cavity-QED-based quantum information processing ${ }^{8,9}$. In particular, a variety of solid-state cavity QED systems have recently been examined ${ }^{15-18}$, not only for the purpose of developing scalable quantum technologies, but also for exploring novel many-body effects inherent to condensed matter. For example, collective $\sqrt{N}$-fold enhancement of light-matter coupling in an $\mathrm{N}$-body system ${ }^{19}$, combined with colossal dipole moments available in solids, compared to traditional atomic systems, is promising for entering uncharted regimes of ultrastrong light-matter coupling. Nonintuitive quantum phenomena can occur in such regimes, including a 'squeezed' vacuum state ${ }^{1}$, the Dicke superradiant phase transition $^{2,3}$, the breakdown of the Purcell effect ${ }^{4}$, and quantum vacuum radiation $^{5}$ induced by the dynamic Casimir effect ${ }^{6,7}$.

Specifically, in a cavity QED system, there are three rates that jointly characterize different light-matter coupling regimes: $g, \kappa$ and $\gamma$. The parameter $g$ is the coupling constant, with $2 g$ being the vacuum Rabi splitting between the two normal modes, the lower polariton (LP) and upper polariton (UP), of the coupled system; see Supplementary Equation (6). The parameter $\kappa$ is the photon decay rate of the cavity; $\tau_{\text {cav }}=\kappa^{-1}$ is the photon lifetime of the cavity, and the cavity $Q=\omega_{0} \tau_{\text {cav }}$ at mode frequency $\omega_{0}$. The parameter $\gamma$ is the nonresonant matter decay rate, which is usually the decoherence rate in the case of solids. Strong coupling is achieved when the splitting, $2 g$, is much larger than the linewidth, $(\kappa+\gamma) / 2$, and ultrastrong coupling is achieved when $g$ becomes a considerable fraction of $\omega_{0}$. The two standard figures of merit to measure the coupling strength are $C \equiv 4 g^{2} /(\kappa \gamma)$ and $g / \omega_{0}$; here, $C$ is called the cooperativity parameter ${ }^{18}$, which is also the determining factor for the onset of optical bistability through resonant absorption saturation $^{20}$. To maximize $C$ and $g / \omega_{0}$, one should construct a cavity QED set-up that combines a large dipole moment (that is, large $g$ ), a small decoherence rate (that is, small $\gamma$ ), a large cavity $Q$ factor (that is, small $\kappa$ ), and a small resonance frequency $\omega_{0}$.

Group III-V semiconductor quantum wells (QWs) provide one of the cleanest and most tunable solid-state environments with quantum-designable optical properties. Microcavity QW-excitonpolaritons represent a landmark realization of a strongly coupled light-condensed-matter system that exhibits a rich variety of coherent many-body phenomena ${ }^{21}$. However, the large values of $\omega_{0}$ and relatively small dipole moments for interband transitions make it impractical to achieve large values of $g / \omega_{0}$ using exciton-polaritons. Intraband transitions, such as intersubband transitions (ISBTs) ${ }^{1}$ and cyclotron resonance $(\mathrm{CR})^{22}$, are much better candidates for accomplishing ultrastrong coupling because of their small $\omega_{0}$, typically in the mid-infrared and terahertz range, and their enormous dipole moments (tens of $e$ - $\AA$ ). Experimentally, ultrastrong coupling has indeed been achieved in GaAs QWs using $\mathrm{ISBTs}^{10,11}$ and $\mathrm{CR}^{12,13}$. In the latter case, a record high value of $g / \omega_{0}=0.87$ has been reported $^{13}$. In all these previous intraband studies of ultrastrong light-matter coupling, however, due to ultrafast decoherence (large $\gamma$ ) and/or lossy cavities (large $\kappa$ ), the value of $C$ remained small; that is, the standard strong-coupling criterion $(C \gg 1)$ was not satisfied.

Here, we simultaneously achieved small $\gamma$ and small $\kappa$ in ultrahigh-mobility two-dimensional electron gases (2DEGs) in GaAs QWs placed in a high-Q one-dimensional (1D) terahertz photonic-crystal cavity (PCC) in a perpendicular magnetic field. We achieved $C>300$ and $g / \omega_{0} \sim 0.1$, observing vacuum Rabi splitting (Rabi oscillations) in the frequency (time) domain. We demonstrated that the influence of this non-perturbative coupling extends even to the region where $\Delta>\omega_{0}$. This can occur only when $g^{2} /\left(\omega_{0} \kappa\right)>1$, which we satisfied through a unique combination of strong light-matter coupling, a small resonance frequency, and a high- $Q$ cavity. Furthermore, we observed a $\sqrt{n_{\mathrm{e}}}$-dependence of 


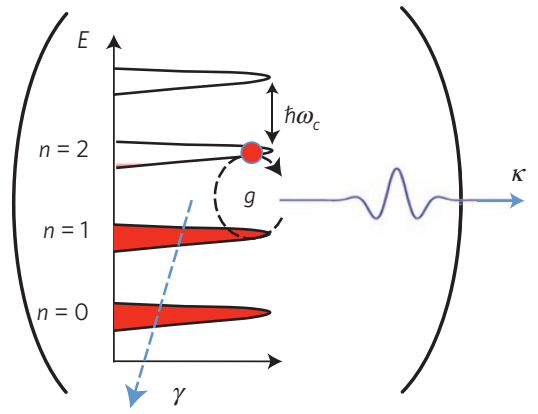

b

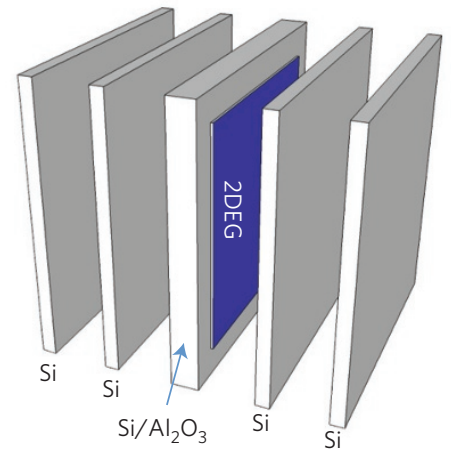

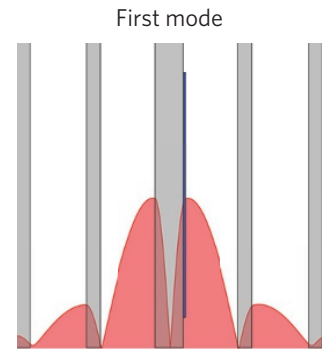

d

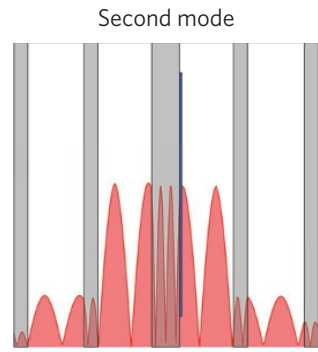

e

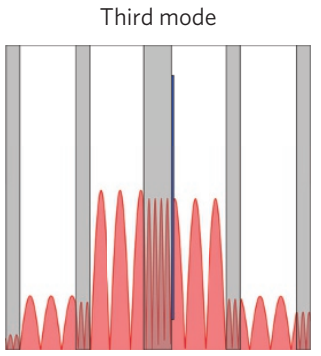

$\mathbf{f}$
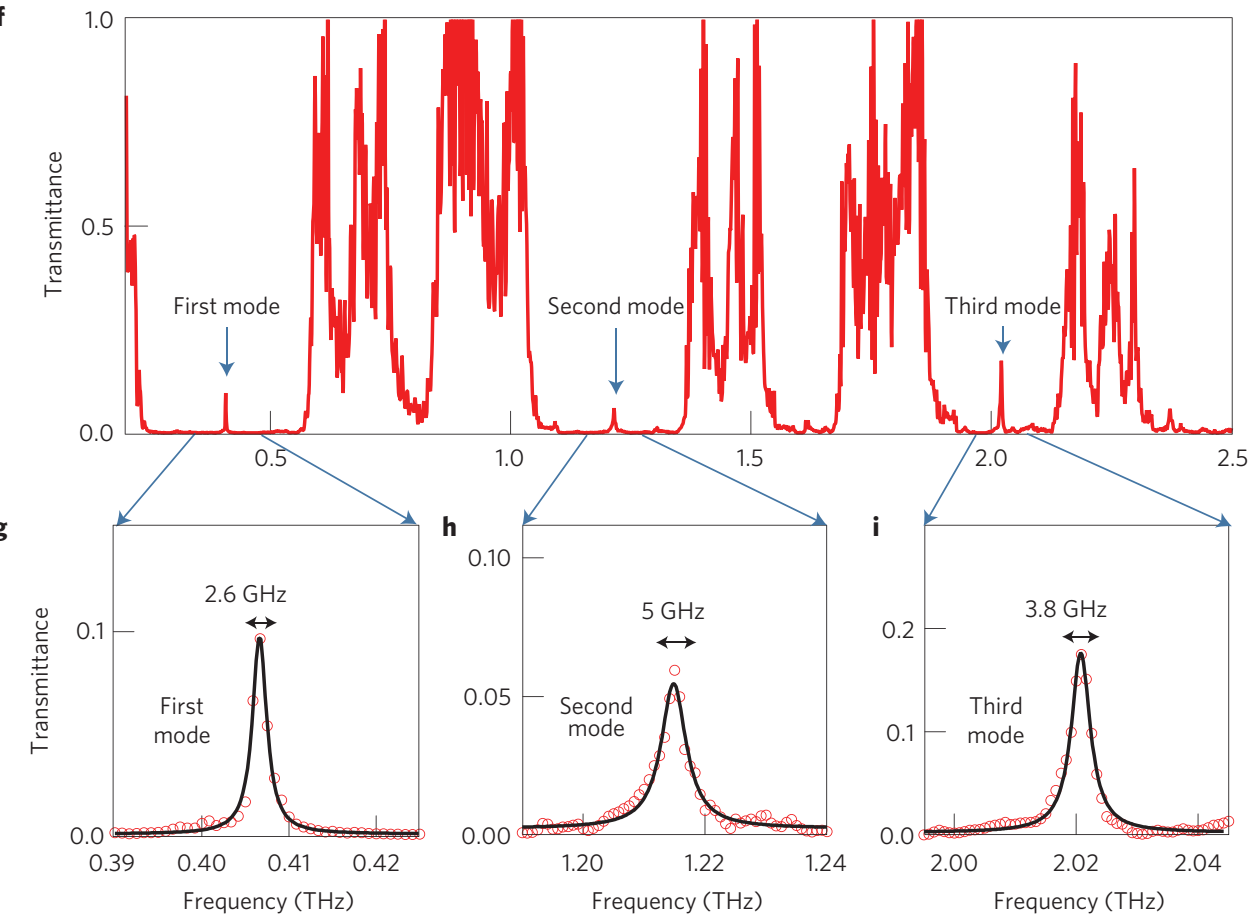

Figure 1 | 1D terahertz photonic-crystal cavity (PCC) with a high-mobility 2DEG. a, Schematic diagram for cyclotron resonance involving two adjacent Landau levels resonantly coupled with a terahertz cavity field. g: light-matter coupling constant, $\kappa$ : photon decay rate, and $\gamma$ : matter decay rate. $\mathbf{b}$, 1D terahertz PCC structure. Two silicon layers are placed on each side of the centre defect layer. The blue part is the transferred 2DEG thin film. $\mathbf{c}-\mathbf{e}$, Calculated electric field amplitude distribution inside the cavity for the first, second, and third cavity modes, respectively. The 2DEG is located at the field maximum for all three cavity modes. f, Experimental power transmittance spectrum for the cavity. Three sharp cavity modes are clearly resolved in the middle of each stopband. g-i, Zoom-in spectra for the three cavity modes, together with Lorentzian fits. The FWHM (Q) values are 2.6 GHz (150), 5 GHz (243) and $3.8 \mathrm{GHz}(532)$ for the first, second and third modes, respectively.

$2 g$ on the electron density $\left(n_{\mathrm{e}}\right)$, signifying the collective nature of light-matter coupling ${ }^{19,23-27}$. A value of $g / \omega_{0}=0.12$ was obtained with just a single QW with a moderate $n_{\mathrm{e}}$. Finally, the previously identified superradiant decay of CR in high-mobility $2 \mathrm{DEGs}^{28}$ was significantly suppressed by the presence of the high- $Q$ terahertz cavity. As a result, we observed ultranarrow polariton lines, yielding an intrinsic CR linewidth as small as $5.6 \mathrm{GHz}$ (or a CR decay time of $57 \mathrm{ps)}$ at $2 \mathrm{~K}$.
High-mobility GaAs 2DEG samples were studied using terahertz time-domain magnetospectroscopy (see Supplementary Section 2). The magnetic field quantized the density of states of the 2DEG into Landau levels. As schematically shown in Fig. 1a, terahertz cavity photons are coupled with the transition between adjacent Landau levels, that is, CR. Figure 1b shows our 1D terahertz PCC design, consisting of two layers of 50- $\mu$ m-thick undoped Si wafers on each side as a Bragg mirror. Thanks to the large contrast 
a

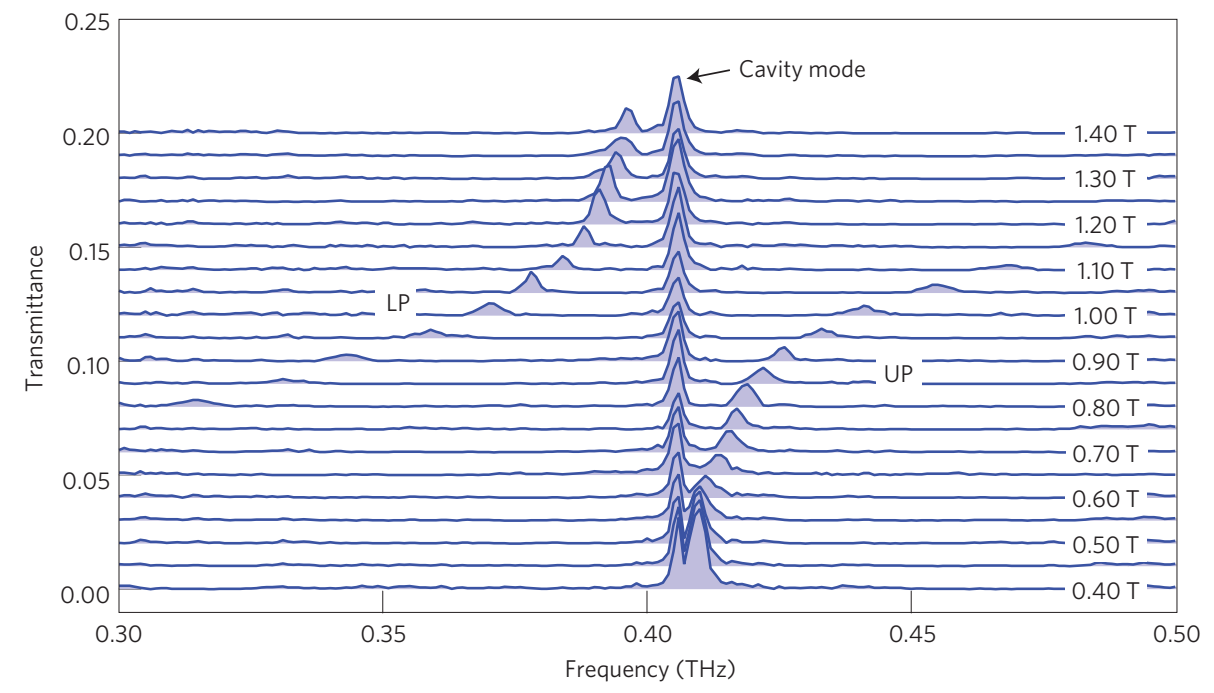

b

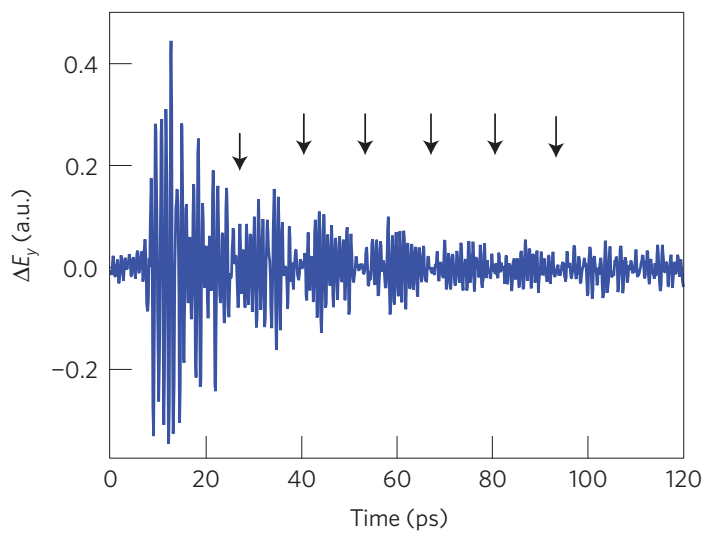

d

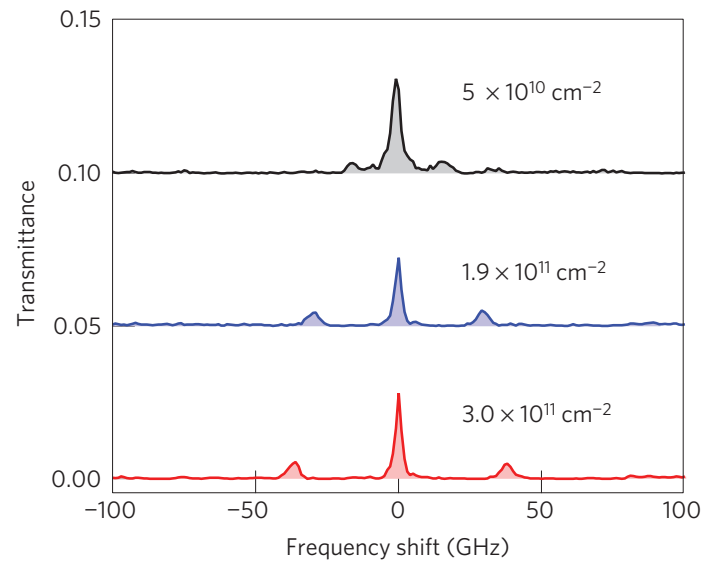

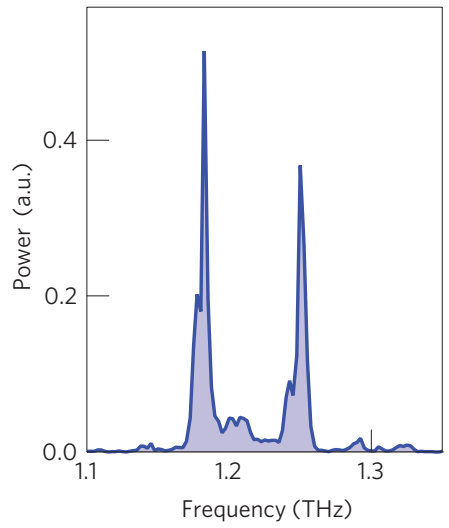

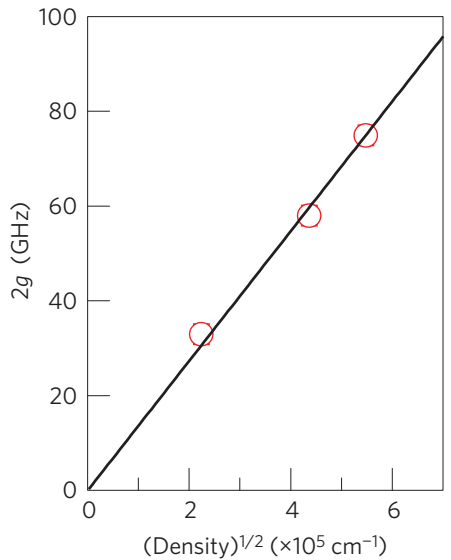

Figure 2 | Observation of collective light-matter coupling in a 2D electron gas in a terahertz photonic-crystal cavity. a, Anticrossing of cyclotron resonance (CR) and the first cavity mode, exhibiting the lower-polariton (LP) and upper-polariton (UP) branches. The central peak due to the cavity mode results from the CR-inactive circularly polarized component of the linearly polarized terahertz beam. Transmission spectra at different magnetic fields are vertically offset for clarity. The magnetic field increases from 0.4 T (bottom) to 1.4 T (top). b, Vacuum Rabi oscillations in the time domain. CR is resonantly coupled with the second cavity mode at 2.975 T. $\Delta E_{y}=E_{y}(+2.975 \mathrm{~T})-E_{y}(-2.975 \mathrm{~T})$ is the measured difference between the transmitted terahertz waveforms taken at $+2.975 \mathrm{~T}$ and $-2.975 \mathrm{~T}$ in the $y$-polarization direction. The residual $\mathrm{CR}$ inactive cavity mode was removed by a numerical notch filter. The beating nodes of the two polaritons are indicated by arrows. c, Frequency-domain spectrum of $\Delta E_{y}$ in $\mathbf{b}$. d, Vacuum Rabi splitting observed for $2 D E G s$ with three different electron densities. CR was resonant with the fundamental cavity mode. e, Square root of $n_{\mathrm{e}}$ dependence of vacuum Rabi splitting, evidencing the collective nature of light-matter coupling.

of refractive index between $\mathrm{Si}$ (3.42 in the terahertz range) and vacuum, only a few layers of Si were required to achieve sufficient cavity confinement of terahertz radiation with high $Q$ values. A substrate-removed 4.5 - $\mu \mathrm{m}$-thick GaAs $2 \mathrm{DEG}$ sample was placed on the central 'defect' layer of the PCC, which was a $100-\mu \mathrm{m}$-thick Si (sapphire) wafer in Cavity 1 (Cavity 2). Calculated electric field distributions inside Cavity 1 are shown in Fig. 1c-e, for the first, second and third cavity modes, respectively. The spatial overlap of the 2DEG and the electric field maximum ensured the strongest light-matter coupling. 
Table 1 | Cavity and matter parameters extracted from experimental data for each cavity mode in the two cavities used.

\begin{tabular}{|c|c|c|c|c|c|c|c|c|c|c|}
\hline Cavity & Mode & $Q$ & $\begin{array}{l}\tau_{\text {cav }} \\
\text { (ps) }\end{array}$ & $\begin{array}{l}B_{r} \\
(T)\end{array}$ & $v$ & $\begin{array}{l}\omega_{0} / 2 \pi \\
(\mathrm{GHz})\end{array}$ & $\begin{array}{l}2 g / 2 \pi \\
(\mathrm{GHz})\end{array}$ & $\begin{array}{l}\kappa / 2 \pi \\
(\mathrm{GHz})\end{array}$ & $\begin{array}{l}\gamma / 2 \pi \\
(\mathrm{GHz})\end{array}$ & $g / \omega_{0}$ \\
\hline 1 & 1 & 183 & 69 & 1.000 & 12.4 & 403 & 74 & 2.6 & 5.6 & 0.09 \\
\hline 1 & 2 & 450 & 57 & 2.975 & 4.2 & 1218 & 66 & 5.0 & $\mathrm{NA}^{\dagger}$ & 0.03 \\
\hline 1 & 3 & 810 & 61 & 4.925 & 2.5 & 2020 & 60 & 3.8 & $\mathrm{NA}^{\dagger}$ & 0.015 \\
\hline 2 & 1 & $>40^{*}$ & - & 0.950 & 13 & 375 & 90 & $<10^{*}$ & $<10^{*}$ & 0.12 \\
\hline 2 & 2 & $>110^{*}$ & - & 2.700 & 4.6 & 1100 & 90 & $<10^{*}$ & $<10^{*}$ & 0.04 \\
\hline
\end{tabular}

Q: quality factor of cavity without $2 D E G, \tau_{\text {cav }}$ : photon lifetime of cavity without $2 D E G, B_{r}$ : resonance magnetic field, $v=n_{\mathrm{e}} h / \mathrm{e} B$ : Landau-level filling factor at $B=B_{r}, \omega_{0}$ : mode frequency, $2 g$ : vacuum Rabi splitting, $\kappa$ : photon decay rate (with 2DEG), and $\gamma$ : nonradiative matter decay rate. ${ }^{*}$ Instrument limited due to insufficient time-scan range. ${ }^{\dagger}$ Not accurately obtainable because of distorted lineshape

(Supplementary Fig. 3).

Figure 1f shows a terahertz transmission spectrum for Cavity 1 , containing a $2 \mathrm{DEG}$, at $4 \mathrm{~K}$. Three photonic band gaps are seen as transmission stopbands. At the centre of each stopband, a sharp cavity mode is observed. As shown in Fig. 1g-i, the full-width at half-maximum (FWHM) values, or $\kappa$, of these cavity modes were $2.6 \mathrm{GHz}, 5.0 \mathrm{GHz}$ and $3.8 \mathrm{GHz}$, corresponding to $Q$ factors of 150 , 243 and 532, respectively; note that these numbers are slightly lower than those for an empty cavity without including the 2DEG, which were 183, 450 and 810 , respectively. These $Q$-factors are one to two orders of magnitude higher than those reported for the terahertz metamaterial resonators employed in previous untrastrongcoupling studies using $2 \mathrm{DEG} \mathrm{CR} \mathrm{CR}^{12,13}$. In the following, experimental data recorded with Cavity 1 are shown. Results obtained with Cavity 2 are described in Supplementary Section 4.

By varying the magnetic field $(B)$, we continuously changed the detuning between the cyclotron frequency $\left(\omega_{\mathrm{c}}=\mathrm{eB} / \mathrm{m}^{*}\right.$, where $m^{*}=0.07 m_{\mathrm{e}}$ is the electron effective mass of GaAs and $m_{\mathrm{e}}=9.11 \times 10^{-11} \mathrm{~kg}$ ) and the cavity mode frequency $\left(\omega_{0}\right): \Delta \equiv \omega_{\mathrm{c}}-\omega_{0}$. Clear anticrossing behaviour, expected for strong coupling, is shown in Fig. 2a for the first cavity mode in Cavity 1. Two polariton branches (LP and UP) were formed through the hybridization of CR and the terahertz cavity photons. The central peak originates from the transmission of the CRinactive circular-polarization component of the linearly polarized incident terahertz beam, which does not interact with the 2DEG and whose position is practically independent of $B$ in this field range. The FWHM of the central peak is thus essentially given by $\kappa$ alone, while that for the LP and UP peaks at $\Delta=0$ is given by $(\kappa+\gamma) / 2$. Therefore, from the $\Delta=0$ spectrum $\left(B_{\mathrm{r}}=1.00 \mathrm{~T}\right.$ for this mode), we determined $\left(2 g, \kappa, \gamma, \omega_{0}\right) / 2 \pi=(74,2.6,5.6,403) \mathrm{GHz}$, yielding $C=360$ and $g / \omega_{0}=0.09$. Parameter values determined in this manner for all modes in both Cavities 1 and 2 are summarized in Table 1, together with cavity parameters and resonance conditions.

As in other cavity QED systems based on atoms and microcavity excitons, vacuum Rabi splitting in the frequency domain can be directly observed as time-domain oscillations ${ }^{23,26,29}$. Experimentally, for an incident terahertz beam linearly polarized in the $x$ direction, we measured the $y$-polarization component, $E_{y}$, of the transmitted terahertz wave, in both positive $(+B)$ and negative $(-B)$ fields, and took the difference, $\Delta E_{y}=E_{y}(+B)-E_{y}(-B)$, to eliminate any background noise; see Supplementary Section 5 for more details. The CR inactive mode was numerically filtered out. As shown in Fig. 2b, the measured $\Delta E_{y}$ signal showed strong beating between the two polariton modes, which can be viewed as coherent repetitive energy exchange between the matter resonance and the cavity photons. At each beating node (indicated by an arrow), energy is stored in the 2DEG CR. The average time separation between two adjacent beating nodes was about 13-15 ps, matching the $2 g$ splitting in the frequency domain; see also Fig. $2 \mathrm{c}$ for the Fourier transform of the time-domain oscillations. The beating lasts for dozens of picoseconds, indicating a long intrinsic CR coherence time.
In analogy to the physics of many-atom light-matter interactions ${ }^{19}$, one crucial question is whether the Rabi splitting observed here is a fully coherent behaviour of a large number of individual electrons in the 2DEG. Figure 2d,e, shows three spectra exhibiting polariton manifestation at $\Delta=0$ for different electron densities $\left(n_{\mathrm{e}}\right)$, when CR is in resonance with the first cavity mode in Cavity 1 . The vacuum Rabi splitting $(2 g)$ between the LP and UP peaks exhibited a square-root dependence on $n_{\mathrm{e}}$ (Fig. 2e), which is strong evidence for collective vacuum Rabi splitting, as observed in atomic gases ${ }^{23}$, spin ensembles $^{24-26}$, and intersubband transitions ${ }^{27}$. This observation validates the notion that billions of $2 \mathrm{D}$ electrons are interacting with a common cavity terahertz photon field in a fully coherent manner. By extrapolation, the vacuum Rabi splitting for CR of a single electron is estimated to be $0.14 \mathrm{MHz}$. It is also worth noting that we should be able to increase the vacuum Rabi splitting further by using multiple layers of a 2DEG with a higher electron density.

The coupled system of Landau-quantized 2D electrons and terahertz cavity photons can be described by the following Hamiltonian $^{22}: \hat{H}_{\text {tot }}=\hat{H}_{\mathrm{CR}}+\hat{H}_{\text {cavity }}+\hat{H}_{\text {int }}+\hat{H}_{\text {dia }}$, where $\hat{H}_{\mathrm{CR}}=\hbar \omega_{\mathrm{c}} \hat{b}^{\dagger} \hat{b}, \hat{H}_{\text {cavity }}=\hbar \omega_{0} \hat{a}^{\dagger} \hat{a}, \hat{H}_{\mathrm{int}}=\hbar g \hat{a}\left(\hat{b}^{\dagger}-\hat{b}\right)+\hbar g \hat{a}^{\dagger}\left(\hat{b}^{\dagger}-\hat{b}\right)$, and $\hat{H}_{\mathrm{dia}}=\left(\hbar g^{2} / \omega_{\mathrm{c}}\right)\left(\hat{a}^{\dagger} \hat{a}^{\dagger}+\hat{a}^{\dagger} \hat{a}+\hat{a} \hat{a}^{\dagger}+\hat{a} \hat{a}\right)$. The first two terms, $\hat{H}_{\text {cavity }}$ and $\hat{H}_{\mathrm{CR}}$, represent, respectively, the energy of the cavity mode at $\omega_{0}$ and the energy of the 2DEG in a magnetic field $B$ with frequency $\omega_{c}$. The operators $\hat{a}$ and $\hat{a}^{\dagger}\left(\hat{b}\right.$ and $\left.\hat{b}^{\dagger}\right)$ are the annihilation and creation operators for cavity photons (collective CR excitations), respectively. The light-matter interaction term, $\hat{H}_{\text {int }}$, with coupling strength $g$ includes counter-rotating terms, $\hbar g\left(\hat{a}^{\dagger} \hat{b}^{\dagger}-\hat{a} \hat{b}\right)$, which are usually neglected under the rotatingwave approximation. Also included in the total Hamiltonian is the diamagnetic term, $\hat{H}_{\text {dia }}$, also known as the $A^{2}$ term because, mathematically, it is proportional to the square of the vector potential $\boldsymbol{A}$ of the light field. The pre-factor $\hbar g^{2} / \omega_{c}$ of the $A^{2}$ term suggests that this term is negligible in the weak-coupling regime, but can have measurable effects when $g$ is a significant fraction of $\omega_{c}$.

Figure 3a presents the best fits to our data with three different Hamiltonians: $\hat{H}_{\mathrm{CR}}+\hat{H}_{\text {cavity }}+\hat{H}_{\text {int }}+\hat{H}_{\text {dia }}$ (full Hamiltonian), $\hat{H}_{\mathrm{CR}}+\hat{H}_{\text {cavity }}+\hat{H}_{\text {int }}$, and $\hat{H}_{\mathrm{CR}}+\hat{H}_{\text {cavity }}+\hbar g\left(\hat{a} \hat{b}^{\dagger}+\hat{a}^{\dagger} \hat{b}\right)$ (the JaynesCummings Hamiltonian, $\left.\hat{H}_{\mathrm{JC}}\right)$. At the optimum fitting, the value of $g / \omega_{0}$ was determined to be 0.09 (for Cavity 1 ). The full CRcavity Hamiltonian provided the best fit, while the other two failed to show the non-negligible blueshift of both polariton modes. Notably, this overall blueshift leads to asymmetric splitting between the two polariton modes with respect to the resonance frequency; this asymmetry is explainable only with the full Hamiltonian. With $g / \omega_{0}=0.1$, the contribution of the $A^{2}$ term is expected to be of the order of $0.01 \omega_{0}$. This $1 \%$ contribution from the $A^{2}$ term is indeed responsible for the observed blueshift (see also Supplementary Fig. 7).

Furthermore, we have evidence that the influence of this non-perturbative coupling extends even to the region where $\Delta>\omega_{0}$, as shown in Fig. 3b,c. This unusually large extent of light-matter hybridization affects the region of negative magnetic fields, where, ordinarily, the UP peak would stay at the cavity 
a

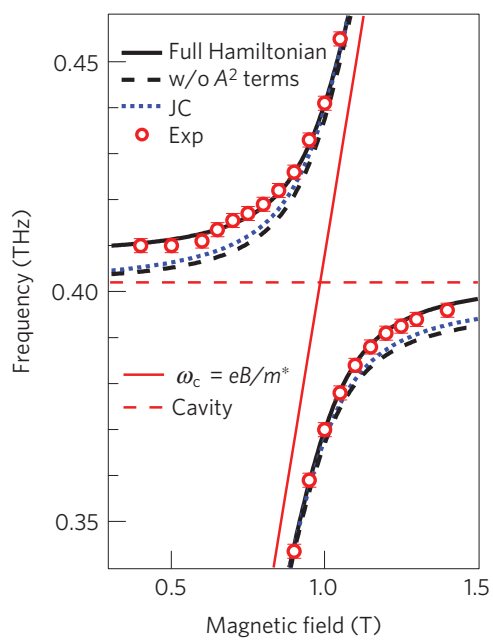

b

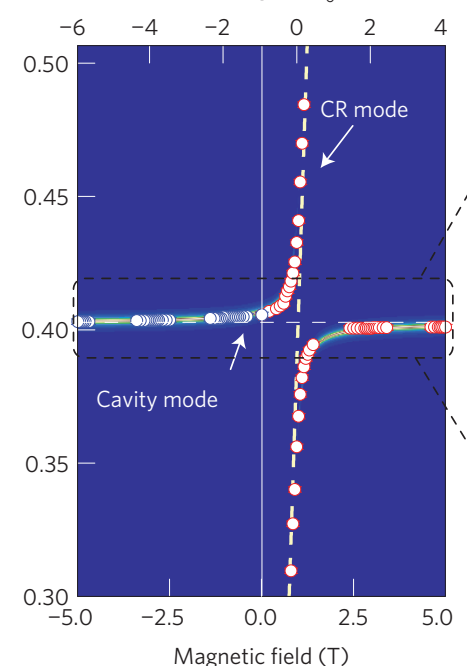

c Detuning $\left(\Delta / \omega_{0}\right)$

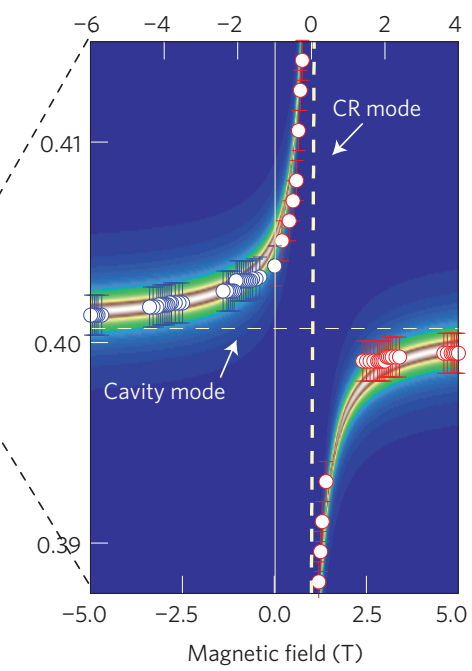

Figure 3 | Theoretical modelling of collective and non-perturbative terahertz light-matter coupling. a, The best fits to our experimental data for the first mode using three different Hamiltonians: the full CR-cavity Hamiltonian (black solid); the full CR Hamiltonian without the $A^{2}$ term (black dashed); the Jaynes-Cummings (JC) Hamiltonian (blue dotted). Only the full CR-cavity Hamiltonian reproduced the experimental data well, suggesting the

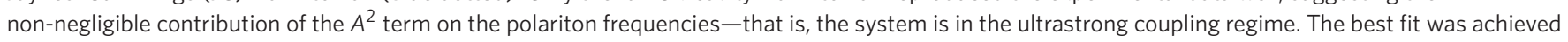
when $g / \omega_{0}=0.09$ for Cavity 1. b,c, Experimental polariton peak positions as a function of magnetic field, together with results of a classical electromagnetic field simulation of terahertz transmission. Red open circles are experimental polariton peak positions for the CRA mode. Blue open circles represent the equivalent CRI mode peak positions at negative magnetic fields. As shown in the zoom-in (c), clear distortion of cavity mode due to light-matter hybridization is even visible at negative magnetic fields, where detuning $\Delta$ is several times larger than $\omega_{0}$. This can occur only when

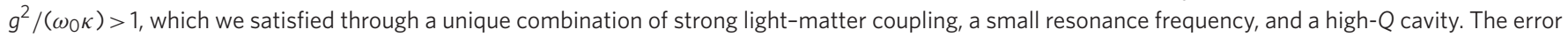
bars represent the frequency resolution defined by the measurement time window.

mode frequency, $\omega_{0}$, independent of $B$. Here, instead, the UP mode is already slightly blueshifted from $\omega_{0}$ even at $B=0$, and then redshifts with increasing $|B|$, asymptotically approaching $\omega_{0}$ at large negative magnetic fields. This counterintuitive behaviour can also be thought of as coupling between the cavity mode and the CR inactive mode at a negative frequency due to the counter-rotating terms in $\hat{H}_{\text {int }}$; see Supplementary Section 7 (Supplementary Fig. 10). The blueshift of the UP peak due to hybridization at finite $\Delta$ can be estimated to be $\left(\sqrt{(2 g)^{2}+\Delta^{2}}-\Delta\right) / 2 \approx g^{2} / \Delta$ around $B=0$, where $\Delta \gg 2 g$. Also, when $B \sim 0$, the UP linewidth $\approx \kappa$. Therefore, for the UP peak to be blueshifted from the cavity mode frequency at $B=0$ (or $\Delta=\omega_{0}$ ), $g^{2} / \omega_{0}>\kappa$ must be satisfied. This condition, $g^{2} /\left(\omega_{0} \kappa\right)>1$, can be met only through a unique combination of strong light-matter coupling, a small resonance frequency, and a high-Q cavity.

Finally, we studied the extracted value of $\gamma$ as a function of temperature. It has previously been shown that the decay of $\mathrm{CR}$ in free space is dominated by collective radiative decay, or superradiance, in ultrahigh-mobility $2 \mathrm{DEG}$, showing a decay rate that is proportional to $n_{\mathrm{e}}$ (ref. 28). This radiative decay mechanism is very strong and dominant at low temperatures, faster than any other phase-breaking scattering processes; thus, CR lines are much broader than expected from the sample mobility. In the present case, however, the emitted coherent $\mathrm{CR}$ radiation cannot readily escape from the high- $Q$ cavity and thus re-excites coherent CR multiple times. Hence, this reversible emission and absorption in a strongly coupled cavity-2DEG system strongly suppresses the superradiant decay, revealing the intrinsic CR decoherence rate, $\Gamma_{\mathrm{CR}}\left(\mathrm{s}^{-1}\right)=\left(\pi \tau_{\mathrm{CR}}\right)^{-1}$. This dramatic suppression of radiative decay is opposite to the Purcell effect in a high- $Q$ cavity expected in the weak-coupling regime, and can only be understood within the framework of strong coupling ${ }^{30}$, where superradiant decay is suppressed by the reversible absorption and emission processes.

Figure $4 \mathrm{a}$ presents temperature-dependent transmission spectra at $\Delta=0$ for the first cavity mode in Cavity 1 . The LP and UP peaks significantly broaden above $20 \mathrm{~K}$, becoming unobservable above $80 \mathrm{~K}$. The central peak, on the other hand, remains essentially unchanged as the temperature increases, serving as an excellent linewidth reference. At each temperature, we determined both $\kappa$ and $\gamma$, using the procedure described earlier (Fig. 2a). As shown in Fig. $4 b$, the CR decay time, $\tau_{\mathrm{CR}}$, measured in the cavity (blue solid circles) is much longer than the superradiance-limited value in free space (red solid circles); at $2 \mathrm{~K}$, the former is $57 \pm 4 \mathrm{ps,}$ while the latter is $10 \mathrm{ps}$. Therefore, the $\tau_{\mathrm{CR}}$ value measured in our high- $Q$ cavity is the intrinsic $C R$ decay time due to nonradiative decay mechanisms (that is, scattering). Also shown in Fig. $4 \mathrm{~b}$ is the temperature dependence of the d.c. momentum scattering time, $\tau_{\text {d.c. }}$, obtained from the electron mobility, $\mu_{\mathrm{e}}=e \tau_{\text {d.c. }} / m^{*}$. Above $20 \mathrm{~K}$, where piezoelectric scattering and polar optical phonon scattering dominate, $\tau_{\mathrm{CR}}$ approaches $\tau_{\text {d.c. }}$. At $2 \mathrm{~K}, \tau_{\mathrm{CR}}$ is still lower than $\tau_{\text {d.c. }}$. How the CR linewidth in a high-mobility 2DEG changes with the magnetic field and temperature is a long-standing question ${ }^{31}$, and a systematic study of 'superradiance-free' CR widths should provide significant new insight.

We have demonstrated collective, coherent, and nonperturbative light-matter coupling between the CR of a 2DEG and terahertz cavity photons in a high-Q $1 \mathrm{D}$ photonic-crystal cavity, with a cooperativity up to 360 . A high- $Q$ cavity is particularly important for cavity-2DEG systems, since it increases not only the cavity photon lifetime but also the matter lifetime via the suppression of superradiant decay. Furthermore, unlike the near-field coupling of metamaterial resonators, our terahertz cavity scheme is applicable to both $2 \mathrm{D}$ and bulk materials, which will allow us to study various strongly correlated systems with collective many-body excitations in the terahertz range; for example, magnetically ordered systems, high-temperature superconductors, and heavy-fermion systems. Hence, our high-Q terahertz cavity-based techniques open a door to a plethora of new possibilities to combine the traditional disciplines of many-body condensed-matter physics and quantum optics of cavity QED. 
a

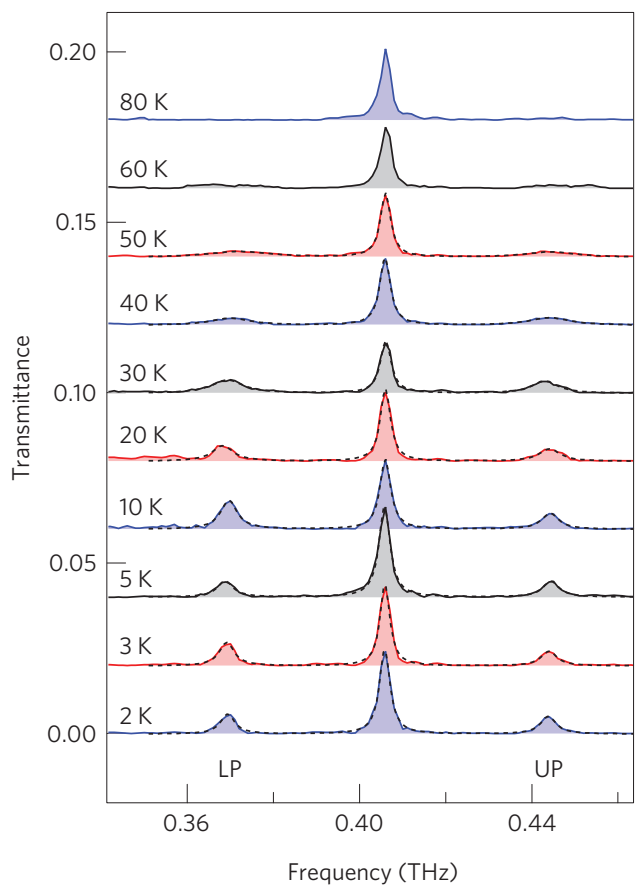

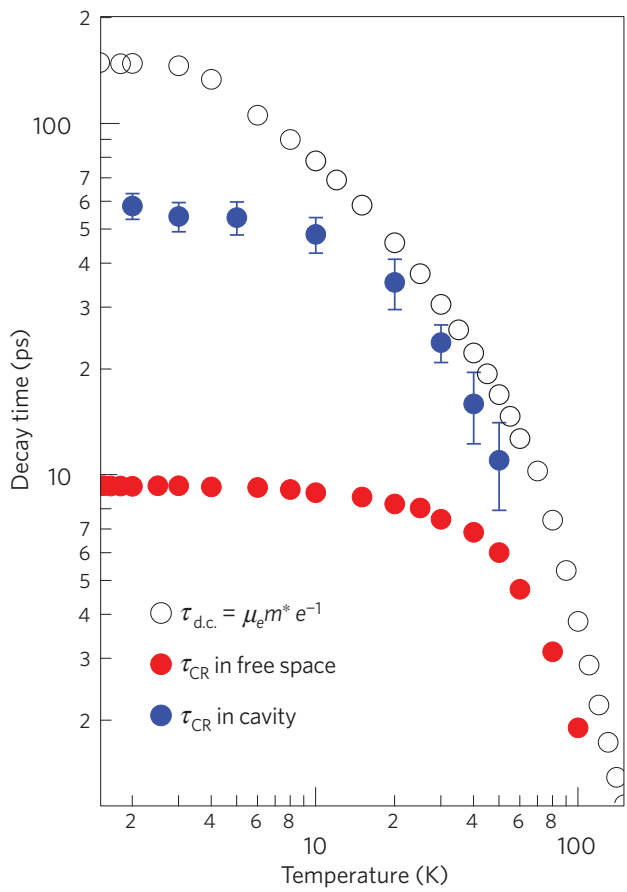

Figure 4 | Observation of ultranarrow CR linewidths due to the suppression of superradiant decay by the high- $Q$ cavity. $\mathbf{a}$, Temperature-dependent spectra showing polariton peaks at zero detuning with the fundamental cavity mode from $2 \mathrm{~K}$ to $80 \mathrm{~K}$ with Lorentzian fits (black dashed lines). Traces are vertically offset for clarity. $\mathbf{b}$, Temperature dependence of the CR decay time $\tau_{\mathrm{CR}}$ measured in free space (red solid circles), $\tau_{\mathrm{CR}}$ measured in the cavity (blue solid circles), and the d.c. momentum scattering time $\tau_{\text {d.c. }}$ (black open circles). The value of $\tau_{\mathrm{CR}}$ in the cavity is $57 \mathrm{ps}$ at $2 \mathrm{~K}$, significantly enhanced from the superradiance-limited $\tau_{\mathrm{CR}}$ of $10 \mathrm{ps}$ in free space. The error bars represent standard deviations.

\section{Methods}

Methods, including statements of data availability and any associated accession codes and references, are available in the online version of this paper.

\section{Received 14 February 2016; accepted 6 July 2016;} published online 22 August 2016

\section{References}

1. Ciuti, C., Bastard, G. \& Carusotto, I. Quantum vacuum properties of the intersubband cavity polariton field. Phys. Rev. B 72, 115303 (2005).

2. Hepp, K. \& Lieb, E. H. On the superradiant phase transition for molecules in a quantized radiation field: the Dicke maser model. Ann. Phys. 76, 360-404 (1973).

3. Wang, Y. K. \& Hioe, F. T. Phase transition in the Dicke model of superradiance. Phys. Rev. A 7, 831-836 (1973).

4. De Liberato, S. Light-matter decoupling in the deep strong coupling regime: the breakdown of the Purcell effect. Phys. Rev. Lett. 112, 016401 (2014).

5. De Liberato, S., Ciuti, C. \& Carusotto, I. Quantum vacuum radiation spectra from a semiconductor microcavity with a time-modulated vacuum Rabi frequency. Phys. Rev. Lett. 98, 103602 (2007).

6. Moore, G. T. Quantum theory of the electromagnetic field in a variable-length one-dimensional cavity. J. Math. Phys. 11, 2679-2691 (1970).

7. Fulling, S. A. \& Davies, P. C. W. Radiation from a moving mirror in two dimensional space-time: conformal anomaly. Proc. R. Soc. Lond. A $\mathbf{3 4 8}$ 393-414 (1976)

8. Imamŏglu, A. et al. Quantum information processing using quantum dot spins and cavity QED. Phys. Rev. Lett. 83, 4204-4207 (1999).

9. Blais, A., Huang, R.-S., Wallraff, A., Girvin, S. M. \& Schoelkopf, R. J. Cavity quantum electrodynamics for superconducting electrical circuits: an architecture for quantum computation. Phys. Rev. A 69, 062320 (2004).

10. Todorov, Y. et al. Strong light-matter coupling in subwavelength metal-dielectric microcavities at terahertz frequencies. Phys. Rev. Lett. 102, 186402 (2009).

11. Geiser, M. et al. Ultrastrong coupling regime and plasmon polaritons in parabolic semiconductor quantum wells. Phys. Rev. Lett. 108, 106402 (2012).

12. Scalari, G. et al. Ultrastrong coupling of the cyclotron transition of a 2D electron gas to a THz metamaterial. Science 335, 1323-1326 (2012).

13. Maissen, C. et al. Ultrastrong coupling in the near field of complementary split-ring resonators. Phys. Rev. B 90, 205309 (2014).
14. Haroche, S. Nobel lecture: controlling photons in a box and exploring the quantum to classical boundary. Rev. Mod. Phys. 85, 1083-1102 (2013).

15. Khitrova, G., Gibbs, H. M., Kira, M., Koch, S. W. \& Scherer, A. Vacuum Rabi splitting in semiconductors. Nat. Phys. 2, 81-90 (2006).

16. Törmä, P. \& Barnes, W. L. Strong coupling between surface plasmon polaritons and emitters: a review. Rep. Prog. Phys. 78, 013901 (2015).

17. Tabuchi, Y. et al. Coherent coupling between a ferromagnetic magnon and a superconducting qubit. Science 349, 405-408 (2015).

18. Viennot, J. J., Dartiailh, M. C., Cottet, A. \& Kontos, T. Coherent coupling of a single spin to microwave cavity photons. Science 349, 408-411 (2015).

19. Dicke, R. H. Coherence in spontaneous radiation processes. Phys. Rev. 93, 99-110 (1954).

20. Bonifacio, R. \& Lugiato, L. A. Dissipative Systems in Quantum Optics (ed. Bonifacio, R.) Theory of optical bistability. Ch. 4, 61-92 (Topics in Current Physics, Springer, 1982).

21. Deng, H., Haug, H. \& Yamamoto, Y. Exciton-polariton Bose-Einstein condensation. Rev. Mod. Phys. 82, 1489-1537 (2010).

22. Hagenmüller, D., De Liberato, S. \& Ciuti, C. Ultrastrong coupling between a cavity resonator and the cyclotron transition of a two-dimensional electron gas in the case of an integer filling factor. Phys. Rev. B 81, 235303 (2010).

23. Kaluzny, Y., Goy, P., Gross, M., Raimond, J. M. \& Haroche, S. Observation of self-induced Rabi oscillations in two-level atoms excited inside a resonant cavity: the ringing regime of superradiance. Phys. Rev. Lett. 51, $1175-1178$ (1983).

24. Amsüss, R. et al. Cavity QED with magnetically coupled collective spin states. Phys. Rev. Lett. 107, 060502 (2011).

25. Tabuchi, Y. et al. Hybridizing ferromagnetic magnons and microwave photons in the quantum limit. Phys. Rev. Lett. 113, 083603 (2014).

26. Zhang, X., Zou, C.-L., Jiang, L. \& Tang, H. X. Strongly coupled magnons and cavity microwave photons. Phys. Rev. Lett. 113, 156401 (2014).

27. Todorov, Y. et al. Ultrastrong light-matter coupling regime with polariton dots. Phys. Rev. Lett. 105, 196402 (2010)

28. Zhang, Q. et al. Superradiant decay of cyclotron resonance of two-dimensional electron gases. Phys. Rev. Lett. 113, 047601 (2014).

29. Norris, T. B. et al. Time-resolved vacuum Rabi oscillations in a semiconductor quantum microcavity. Phys. Rev. B 50, 14663-14666 (1994).

30. Tignon, J. et al. From Fermi's golden rule to the vacuum Rabi splitting: magnetopolaritons in a semiconductor optical microcavity. Phys. Rev. Lett. 74, 3967-3970 (1995).

31. Andreev, I. V., Muravev, V. M., Belyanin, V. N. \& Kukushkin, I. V. Measurement of cyclotron resonance relaxation time in the two-dimensional electron system. Appl. Phys. Lett. 105, 202106 (2014). 


\section{Acknowledgements}

We thank A. Chabanov, H. Pu and A. Belyanin for useful discussions.

J.K. acknowledges support from the National Science Foundation

(Grant No. DMR-1310138). This work was performed, in part, at the Center for Integrated Nanotechnologies, a US Department of Energy, Office of Basic Energy Sciences user facility. Sandia National Laboratories is a multiprogram laboratory managed and operated by Sandia Corporation, a wholly owned subsidiary of Lockheed Martin Corporation, for the US Department of Energy's National Nuclear Security Administration under Contract No. DE-AC04-94AL85000. The work at Sandia was supported by the US Department of Energy, Office of Science, Materials Sciences and Engineering Division. Growth and characterization completed at Purdue by J.D.W. was supported by the Department of Energy, Office of Basic Energy Sciences, Division of Materials Sciences and Engineering under Award No. DE-SC0006671.

M.J.M. acknowledges additional support from the W. M. Keck Foundation and Microsoft Research.

\section{Author contributions}

Q.Z. designed and fabricated the terahertz photonic-crystal cavities, performed all measurements, analysed all experimental data, and performed all theoretical simulations, under the supervision and guidance of J.K.; M.L. and X.L. assisted Q.Z. with the simulations and measurements. J.L.R., W.P., J.D.W. and M.J.M. provided the 2DEG samples. Q.Z. and J.K. wrote the manuscript. All authors discussed the results and commented on the manuscript.

\section{Additional information}

Supplementary information is available in the online version of the paper. Reprints and permissions information is available online at www.nature.com/reprints.

Correspondence and requests for materials should be addressed to J.K.

\section{Competing financial interests}

The authors declare no competing financial interests. 


\section{Methods}

We used polarization-resolved time-domain terahertz magnetospectroscopy to study the 2DEG samples in terahertz cavities placed inside a split-coil

superconducting magnet. Our laser source was a Ti:sapphire regenerative amplifier $(1 \mathrm{kHz}, 775 \mathrm{~nm}, 200 \mathrm{fs}$, Clark-MXR, Inc., CPA-2001). The nonlinear crystals used for both terahertz generation and detection were 1-mm-thick $\langle 110\rangle$-oriented zinc telluride (ZnTe). We studied two samples of modulation-doped GaAs 2DEG with a single quantum well. Sample 1 had density and mobility values of $1.9 \times 10^{11} \mathrm{~cm}^{-2}$ and $2.2 \times 10^{6} \mathrm{~cm}^{2} \mathrm{~V}^{-1} \mathrm{~s}^{-1}$, respectively, in the dark, while after illumination at $4 \mathrm{~K}$ they changed to $3.1 \times 10^{11} \mathrm{~cm}^{-2}$ and $3.9 \times 10^{6} \mathrm{~cm}^{2} \mathrm{~V}^{-1} \mathrm{~s}^{-1}$; Sample 2 had $5 \times 10^{10} \mathrm{~cm}^{-2}$ and $4.4 \times 10^{6} \mathrm{~cm}^{2} \mathrm{~V}^{-1} \mathrm{~s}^{-1}$ as the density and the mobility, respectively. The GaAs substrate was removed by selective etching. See Supplementary Information for more experimental details.

Data availability. The data that support the plots within this paper and other findings of this study are available from the corresponding author upon reasonable request. 